\title{
A retrospective study of bladder cancer and the impact of age, sex and smoking habits with related clinicopathological correlations in the tribal population of Bankura,WB,India.
}

\author{
Dr.Debkumar Ray ${ }^{1}$, Dr.Ramkrishna Mondal ${ }^{2}$,Dr.Santanu Achryya ${ }^{3}$, \\ Dr.Sumitava De ${ }^{4}$,Dr.Srikrishna Mondal ${ }^{5}$, \\ 1.Assistant Professor, Department of Biochemistry, B.S.Medical College, Bankura, WB \\ 2.Associate Professor, Department of Surgery, B.S.Medical College,Bankura,WB \\ 3.Assistant Professor, Department of Radiotherapy, B.S.Medical College,Bankura,WB \\ 4.RMO cum Clinical Tutor, Depatrment of Radiotherapy, B.S.Medical College,Bankura,WB \\ 5.Professor,Depatrment of Radiotherapy B.S.Medical College,Bankura, WB
}

\begin{abstract}
Aim: To determine the impact of age, gender and smoking on the clinicopathological characteristics of histologically confirmed bladder cancer in the tribal population of Eastern India.

Materials and Methods: From January 2008 to December 2012, records of patients with bladder cancer were evaluated for age and gender at presentation, clinical symptoms, cystoscopic finding, history of smoking, and histopathological characteristics. A total of 264 patients were identified from the hospital information system and the case files of patients.

Results: The incidence of $4.85 \%$ among the total cancer patients is slightly higher than the national average . The median age was 57 years old and the male to female ratio was 6.2:1. Most common presenting symptom was painless hematuria (91\%). Transitional cell carcinoma (TCC) was the most common histological variety $(96.59 \%)$. A total of $38.25 \%$ of the patients had muscle invasive disease at the time of presentation. Low grade tumors were higher in females and non-smokers being statistically significant.

Conclusion: TCC is the predominant cancer, with significant male preponderance among tribal populations in Eastern Indians. Younger-aged, females and non smoker patients have low-grade disease. Hematuria is the most common presentation and greater awareness is needed not to overlook bladder cancer.
\end{abstract}

Keywords: Bladder cancer. transitional cell carcinoma, smoking

\section{Introduction}

Bladder cancer is one of the most common urological malignancies. As per the Indian cancer registry data in men, it is the ninth most common cancer accounting for 3.9\% of all cancer cases. [1] The median age at diagnosis was 69 years old for males and 71 years old for females. [2] It is three times more common in men than in women, and $90 \%$ of the bladder tumors are transitional cell carcinoma (TCC). [3] Age, gender, and racial factors all affect the survival and prognosis of patients with bladder cancer. [4] A total of 40 to $45 \%$ of newly diagnosed bladder cancers are high-grade lesions, more than half of which are muscle invasive at the time of diagnosis. [5] To our knowledge, very few studies have been presented to describe the demographic data of bladder cancer in India, particularly in the tribal populations of eastern India. Herein, we present the clinicopathological characteristics of bladder cancer with an analysis of the impact of age, gender and smoking on tumor biology.

\section{Materials and Methods}

From January 2008 to December 2012, data from all the patients with bladder cancer entered on the hospital information system and in the case record files were evaluated for age, gender, symptoms, history of smoking, and histopathological characteristics at the time of presentation.

A total of 264 patients presented with bladder cancer whose complete records were available, which formed the study group.

When bladder cancer was identified on a cystoscopy, the location, number, and nature of the disease were recorded. As most of the patients were referred to us with the diagnosis, there was no consistent record on urine cytology. A transurethral resection of the bladder tumor (TURBT) was performed using Glycine (1.5\%) as an irrigant. A deep biopsy was taken separately to include the detrusor muscle. A random biopsy was not performed as per the protocol and only suspicious lesions were biopsied.

The new World Health Organization (WHO) and International Society of Urological Pathology (ISUP) classifications were used for pathological grading. Data were recorded as Ta for papillary, urothelial-confined carcinoma, T1 for lamina-invasive carcinoma, and T2 for muscle-invasive carcinoma. In this study, for the 
purpose of statistical analysis, Grade 1 and Grade 2 were classified as low-grade and Grade 3 was classified as high-grade. The biopsy specimen in which there was no evidence of detrusor muscle was recorded separately.

Data were analysed using SPSS software, Version 16 (SPSS, Chicago, IL, USA) and the Chi-square test was used to compare the variables. A P-value of less than 0.05 was taken as significant.

\section{Results}

In our study we found 264 bladder cancer patients among the total number of patients of 5436 whose complete records were available. The incidence of $4.85 \%$ among the total cancer patients is slightly higher than the national average of $3.9 \%$. Of the 264 patients, $91 \%$ presented with painless hematuria. The next most common symptoms were dysuria and frequency. TCC was the most common variant seen in $96.59 \%$ of the patients [Table 1]. $38.25 \%$ of the patients had muscle-invasive disease at the time of presentation while the remaining $61.75 \%$ had nonmuscle- invasive bladder carcinoma in which $37.1 \%$ had p Ta and $24.6 \%$ had p T1.

Overall, $46.2 \%$ of the patients had low-grade disease. Detrusor muscle was not evident in $36.36 \%$ of the patients in their biopsy specimen.

The median age at presentation was 57 years old (range: 26- 74 years old). The age- wise pathological distribution of bladder tumors is shown in [Table 1].

Only 5.3\% (14 of 264) of the patients presented with bladder cancer at younger than 40 years of age. Low-grade cancer was found more commonly in patients younger than 60 years old as compared with patients older than 60 years old which was statistically significant $(52.6 \%$ vs. $39.5 \%$; P-value $=0.03)$.

Although not statistically significant, nonmuscle- invasive bladder tumors (Ta and T1) were relatively more common in the younger age group $(65.92 \%$ vs. $57.8 \%)$ and muscle-invasive tumors were more common in the group of patients who were older than 60 years old $(42.9 \%$ vs. $34 \%$; $p$ value $=0.16$. The male to female ratio was $6.2: 1$. A total of $76.2 \%$ of the males and $45.9 \%$ of the females with bladder cancer smoked or had an intake of tobacco in some form. . Low-grade cancer was found more commonly in smokers than non smokers $(68.95 \%$ vs. $43.24 \%$; P-value $=0.0002)$. Low-grade cancer was found more commonly in females than males $(64.86 \%$ vs. $43.17 \%$; P-value $=0.03$ ). [Table 2], [Table 3].

Of the 264 patients, 93 patients were in the high-risk category, which included TaHG, T1LG, and T1HG. Of the 93 patients, 75 patients received Bacillus Calmette-Gurrin (BCG) as induction therapy. Each patient received BCG weekly for 6 weeks. Of the 101 patients with muscle-invasive bladder cancer, 46 patients underwent a radical cystectomy and standard pelvic lymph node dissection with urinary diversion. A total of 6 patients died during the mean follow-up of 36 months (5-54 months).

\section{Discussion}

According to the Delhi Cancer Registry, in 2003, bladder cancer was the 6 most common cancer, surpassed in frequency only by cancers of the lung, larynx, tongue, prostate, and esophagus. Fifteen percent of all tobaccorelated cancers are bladder cancers. Bladder cancerpresents with painless hematuria in $80-85 \%$ of the patients. However, in reality, nearly all patients with cystoscopically detectable bladder cancer have at least microhematuria if enough urine samples are tested. In our study, $91.0 \%$ of the patients presented with gross hematuria. This high incidence may be due to a lack of screening for microscopic hematuria in the form of dipstick or flexible cystoscopy, even in high-volume centers.

TCC is the most common variant accounting for $90 \%$ of bladder cancer in the world literature. [3] Considerable variability is noted in the prevalence of squamous cell carcinoma (SCC) of the bladder in different parts of the world. SCC accounts for only 1\% of bladder cancers in England, 3-7\% in the United States, but as much as 75\% in Egypt. Adenocarcinoma (AC) accounts for less than $2 \%$ of bladder cancer. [2] In our study, $96.59 \%$ of the patients had TCC, whereas SCC and AC accounted for $3.03 \%$ and $0.38 \%$ of the patients, respectively.

Cigarette smokers have at least a four times higher incidence of bladder cancer. [6],[7] This risk has been observed in both the genders.

Former cigarette smokers have a reduced incidence of bladder cancer compared with active smokers. [8] When comparing p53 mutations in the bladder cancer of smokers with those in the bladder cancer of patients who have never smoked, differences in the types or sites of mutations have not been seen, although a higher number of mutations occurred in smokers. This suggests that smoking might increase the amount of mutations in urothelial cells without necessarily directing the site or type of mutation. [9] In our study, 71.97\% (190 of 264) of the patients were smokers. In one series reported on bladder cancer, the percentage of male smokers was $50 \%$ and $31 \%$ for female smokers. [10] Contrary to this, the incidence of smoking or intake of tobacco in any form in our patients with bladder cancer was much higher among males compared with females (76.2\% vs. $45.9 \%)$. Bladder cancer is rare in people younger than 50 years of age, even though it can occur at any age. The incidence of cancer increases directly with age with the median age at diagnosis of around 70 years for each gender. [2] In our study, the median age was 57 years old. 
Younger individuals present more frequently with low-grade and low-stage tumors than their elderly counterparts [11] and behave in an indolent fashion. [12] The same has been observed in our study. This is contrary to the common belief in malignancy that biological behaviour of a cancer is more aggressive in younger age groups. This fact is underplayed in the literature and research should be carried out to find out the reasons for this. However, it has been observed that genetic alterations that are frequently seen in older adults are extremely rare in young patients. Urothelialneoplasms in children and young adults appear to be biologically distinct and lack genetic instability in most cases. [13] In our study, the male to female ratio was 6.2:1, which is different from what is reported in the literature, i.e., 3:1-5:1. [14] A much higher incidence of tumors was detected in males compared with females. Possible explanations for the excessive risk in men include environmental and dietary exposures not yet identified and innate sexual characteristics such as anatomic differences, urination habits, or hormonal factors. [15],[16] Compared with data available from the west, a disproportionate decrease of tumor incidences in females can be due to the decreased exposure to industrial carcinogens as fewer number of women work outside the home. It is also observed that there is a decreased exposure of females to tobacco intake, which has been the major predisposing factor for bladder cancer. Also, females tend to present to the hospital less because of social reasons. Incidentally non-smoker women have larger and more aggressive tumors with a frequency of muscle-invasive disease than male non-smokers.[17] This has also reflected in our study as Low-grade cancer was found more commonly in smokers than non smokers $(68.95 \%$ vs. $43.24 \%$; P-value $=0.0002)$.

In our series, $36.36 \%$ of the patients did not show evidence of detrusor muscle in their biopsy specimen. This is in concordance with the literature, in which the detrusor muscle was absent in 40-51\% TUR specimens. [18] Not including the detrusor muscle in the biopsy specimen may lead to understaging in many patients.

\section{Conclusion}

In the Indian population, bladder cancer is predominantly TCC and more than $90 \%$ of the patients present with painless hematuria. Male preponderance is much more frequent in Indians than in other races. Younger patients present with low-grade disease for which further studies are required to understand this biological difference. Awareness is needed among the public and treating physicians as they tend to neglect the symptoms of hematuria, resulting in an advanced stage of bladder cancer at presentation.

\section{References}

[1]. Kurkure AP. Cancer incidence and patterns in urban Maharashtra. Consolidated report of the population based cancer registries, Year 2001 .

[2]. Lynch CF, Cohen MB. Urinary system.Cancer 1995; 75:316-29.

[3]. Rabbani F, Cordon-Cardo C. Mutation of cell cycle regulators and their impact on superficial bladder cancer. UrolClin North Am 2000; 27:83-102.

[4]. Madeb R, Messing EM. Gender, racial and age differences in bladder cancer incidence and mortality.UrolOncol 2004; 22:86-92.

[5]. Messing EM, Young TB, Hunt VB, Gilchrist KW, Newton MA, Bram LL, et al. Comparison of bladder cancer outcome in men undergoing hematuria home screening versus those with standard clinical presentations. Urology 1995; 45:396-7.

[6]. Morrison AS. Advances in the etiology of urothelial cancer.UrolClin North Am 1984; 11:557-66.

[7]. Burch JD, Rohan TE, Howe GR, Risch HA, Hill GB, Steele R, et al. Risk of bladder cancer by source and type of tobacco exposure: A case-control study. Int J Cancer 1989; 44:622-8.

[8]. Augustine A, Hebert JR, Kabat GC, Wydner EL. Bladder cancer in relation to cigarette smoking. Cancer Res 1988; 48:4405-8.

[9]. Spruck CH 3rd, Rideout WM 3rd, Olumi AF, Ohneseit PF, Yang AS, Tsai YC, et al. Distinct pattern of p53 mutations in bladder cancer: Relationship to tobacco usage. Cancer Res 1993; 53:1162-6.

[10]. Wydner EL, Goldsmith K. The epidemiology of bladder cancer: A second look. Cancer 1977; 40:1246.

[11]. Wan J, Grosman HB. Bladder carcinoma in patients age 40 years or younger.Cancer 1989; 64:178-81.

[12]. Linn JF, Sesterhann I, Mostofi FK, Schoenberg M. The molecular characteristics of bladder cancer in young patients.J Urol 1998; 159:1493-6.

[13]. Wild PJ, Giedl J, Stoehr R, Junker K, Boehm S, Van Oers JM, et al. none Genomic aberrations are rare in urothelialneoplasms of patients 19 years or younger. J Pathol 2007; 211:18-25.

[14]. Jemal A, Murray T, Ward E, Samuels A, Tiwari RC, Ghafoor A, et al. Cancer statistics, 2005. CA Cancer J Clin 2005; 55:10-30.

[15]. Hartge P, Harvey EB, Linehan WM, Silverman DT, Sullivan JW, Hoover RN, et al. Unexplained excess risk of bladder cancer in men. J Natl Cancer Inst 1990; 82:1636-40.

[16]. Horn EP, Tucker MA, Lambert G, Silverman D, Zametkin D, Sinha R, et al. A study of gender-based cytochrome P450 1A2 variability: A possible mechanism for the male excess of bladder cancer. Cancer Epidemiol Biomarkers Prev 1995; 4:529-33.

[17]. 17.Goonewardena, SA De Silva WA, De Silva MV et al. Bladder cancer in Sri Lanka: experience from a tertiary referral center. Int. Jour. Uro 2004 Nov; 11(11):969-72.

[18]. .Maruniak NA, Takezawa K, Murphy WM. Accurate pathological staging of urothelialneoplasms requires better cystoscopic sampling. J Urol 2002; 167:2404-7. 
TABLE 1. ). The age- wiseClinicopathological distribution of bladder tumors.

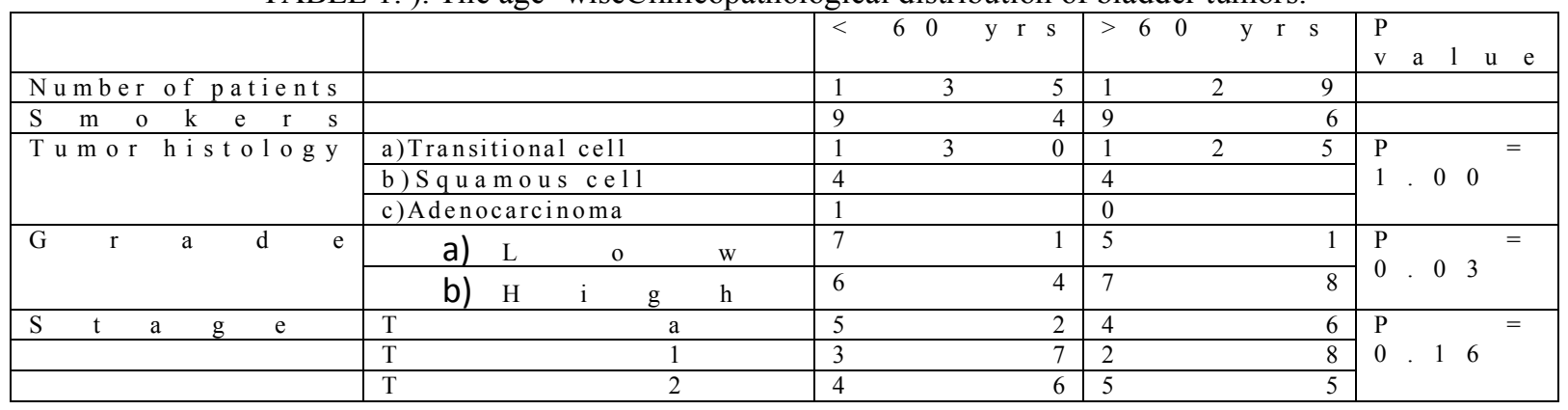

TABLE 2).The gender- wiseClinicopathological distribution of bladder tumors.

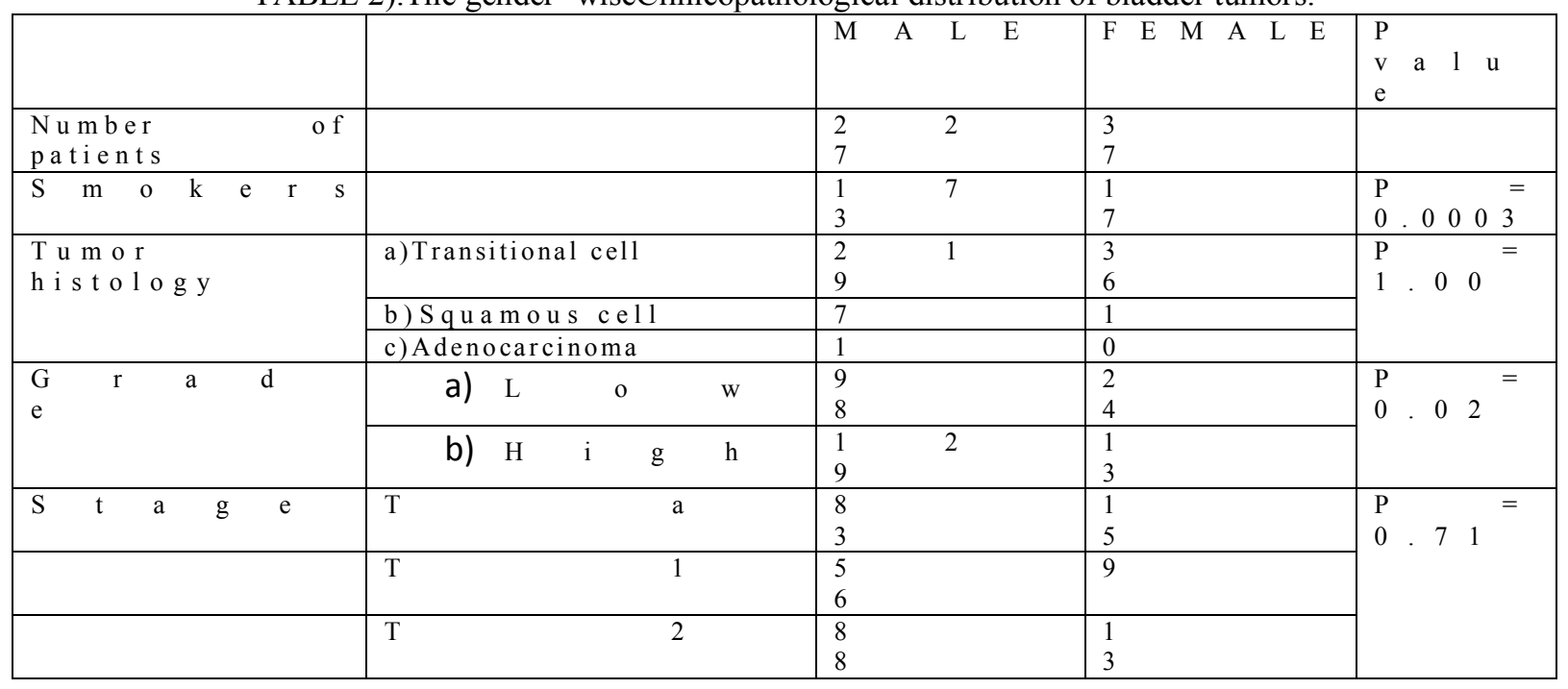

TABLE 3). The pathological distribution of bladder tumors based on smoking habit.

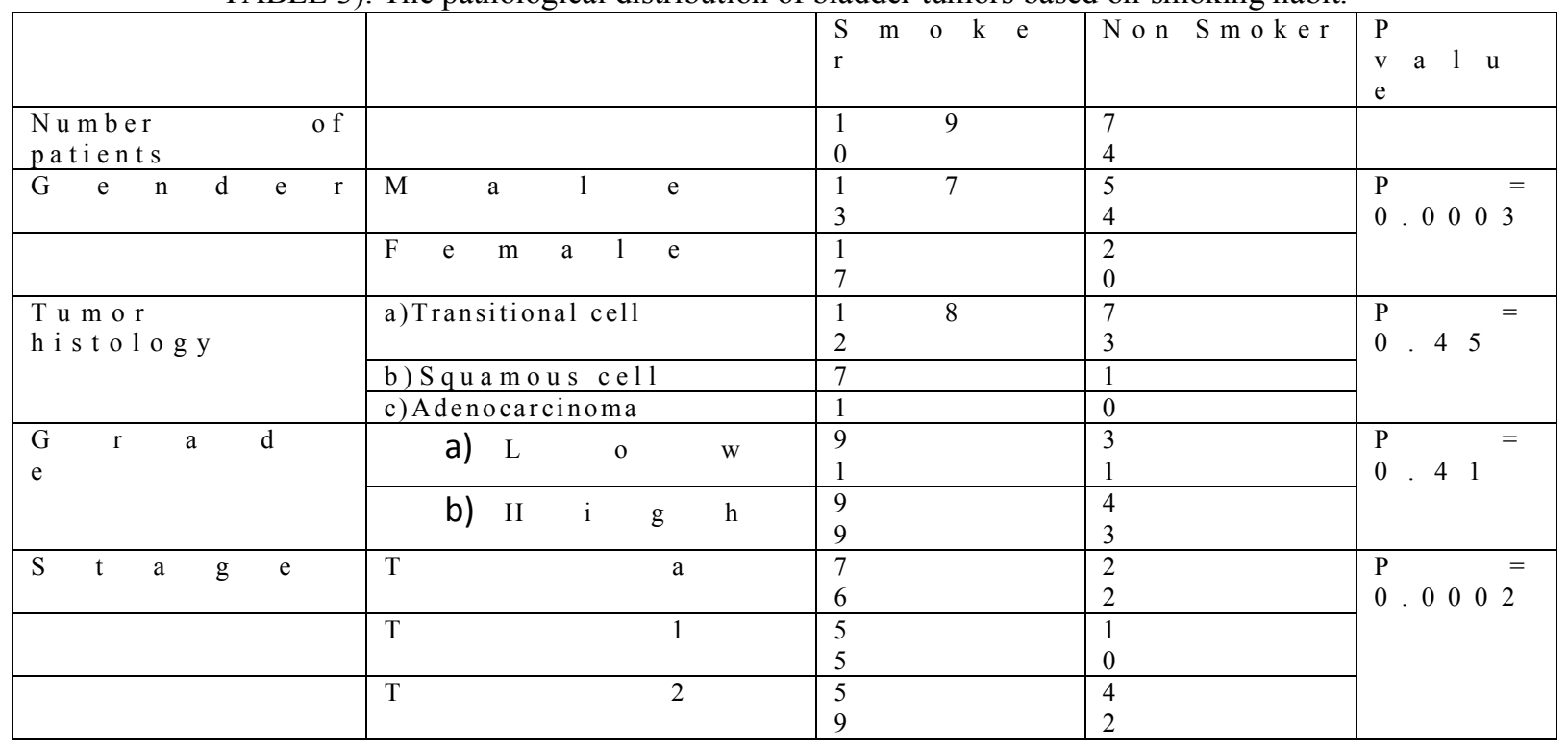

\section{Legends:}

[1]. The age- wise Clinicopathological distribution of bladder tumors.

[2]. The gender- wiseClinicopathological distribution of bladder tumors.

[3]. The Clinicopathological distribution of bladder tumors based on smoking habit. 